\title{
An unusual presentation of generalized aggressive periodontitis with multiple impacted supernumerary teeth
}

Arif Salman ${ }^{1}$

Archana Meethil ${ }^{2}$

\begin{abstract}
Aggressive periodontitis is a rare condition that progresses rapidly but affects only a small percentage of population. Most of the cases are familial. The presence of supernumerary teeth is also rather rare and often familial. Therefore, a concomitant presentation of aggressive periodontitis and supernumerary teeth in an individual has generated a great interest among clinicians. Here, we report a rare nonsyndromic case of generalized aggressive periodontitis with multiple impacted supernumerary teeth. (Eur J Dent 2012;6:335-339)
\end{abstract}

Key words: Aggressive periodontitis; multiple supernumerary teeth; supernumerary teeth; nonsyndromic case; dental anomalies.

\section{INTRODUCTION}

Aggressive periodontitis is a group of rare, rapidly progressive, and severe forms of periodontitis that clinically present at an early age and often show a familial pattern. Aggressive periodontitis is characterized by accelerated loss of attachment and bone destruction in otherwise systemically healthy patients, and the amount of microbial deposits does not correspond to the severity of the disease. $^{1}$

\footnotetext{
1 Department of Periodontology and Implant dentistry, Sree Balaji Dental College and Hospital, Chennai, INDIA

2 Department of Periodontology, SRM Kaatankulathur Dental College, Chennai, INDIA

- Corresponding author: Department of Periodontology and Implant dentistry, Sree Balaji Dental College and Hospital, Velachery Main Road, Narayanapuram, Pallikaranai, Chennai - 600100, India.

Tel: +919884243529

Email: drarifsalmandgmail.com
}

Supernumerary teeth are additional teeth beyond the normal full complement in the primary or permanent dentition. Supernumerary teeth are usually more frequent in men than in women, with a prevalence of $0.3-0.8 \%$ in the primary series and $0.1-3.8 \%$ in the permanent series. Supernumerary teeth can occur as isolated or multiple teeth in both jaws. However, only 1 supernumerary tooth or more than 2 supernumerary teeth show greater predilection for the anterior region of the maxilla than for the mandible; on the other hand, multiple supernumerary teeth (more than 5) are often observed in the mandibular premolar region. ${ }^{2}$

Multiple supernumerary teeth are very rarely observed, and in most cases, they are associated with syndromes such as Gardner's syndrome, cleidocranial dysplasia, and cleft lip and palate. ${ }^{3}$ However, a concomitant presentation of aggressive periodontitis and supernumerary teeth is un- 
common. ${ }^{4}$ Here, we report a nonsyndromic case of generalized aggressive periodontitis with multiple impacted supernumerary teeth.

\section{CASE REPORT}

A 20-year-old normal, healthy man complained of mobility of the left upper front tooth that had persisted for 6 months. One year before his presentation, he had visited an orthodontist, who referred him to a periodontist. He did not have a family history of tooth mobility, his medical history was noncontributory, and he was a nonsmoker.

Intraoral examination showed a full complement of permanent teeth, except a clinically missing right permanent mandibular third molar. The gingiva appeared red, showed an altered contour, and lacked stippling. The anterior maxillary and mandibular teeth showed minimal deposits of plaque and calculus. Gingival probing elicited bleeding and showed the presence of deep periodontal pockets. Many teeth showed Millers grade I mobility, whereas the left maxillary central incisor showed grade III mobility. A few anterior teeth showed pathological migration (Figure 1). Most of

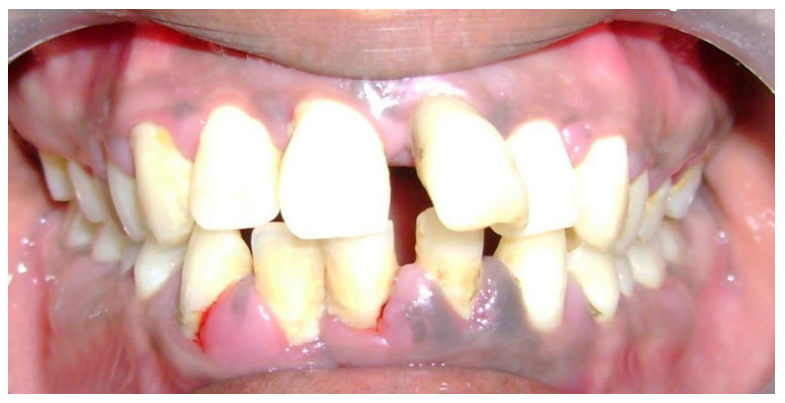

Figure 1. Initial presentation showing gingival inflammation, papillary enlargement and distolabial migration of permanent left upper central incisor. the molars showed furcation involvement. Panoramic radiography showed generalized, mild-tomoderate horizontal bone loss; however, vertical bone defects were restricted to only certain teeth. Panoramic radiography also showed 6 impacted supernumerary teeth; 2 supplemental premolars in the maxilla and 4 distomolars, 1 in each quadrant (Figure 2). Because multiple supernumerary teeth are often associated with certain syndromes, our patient was clinically examined to rule out these syndromes. The patient's sibling and parents were also examined clinically and radiographically, but they did not show any pathological alterations, including supernumerary teeth. According to the clinical and radiological findings, the patient was diagnosed with generalized aggressive periodontitis with multiple impacted supernumerary teeth.

The patient was informed about his oral condition, and a comprehensive treatment plan was presented to him. The left maxillary permanent central incisor was extracted, and full-mouth scaling and root planning were performed under local anesthesia. This procedure was supplemented with systemic antibiotic therapy consisting of amoxicillin (500 $\mathrm{mg}$ tid for 7 days) and metronidazole (400 mg tid for 7 days). After 6 weeks, the patient was again examined clinically; because of the persistence of periodontal lesions, he was advised to undergo flap surgery to provide access to instrumentation and regenerative therapies. Sufficient healing was achieved after the surgical management; next, a fixed partial denture made of porcelain fused to metal was fabricated and inserted in relation to teeth 11,21, and 22. After

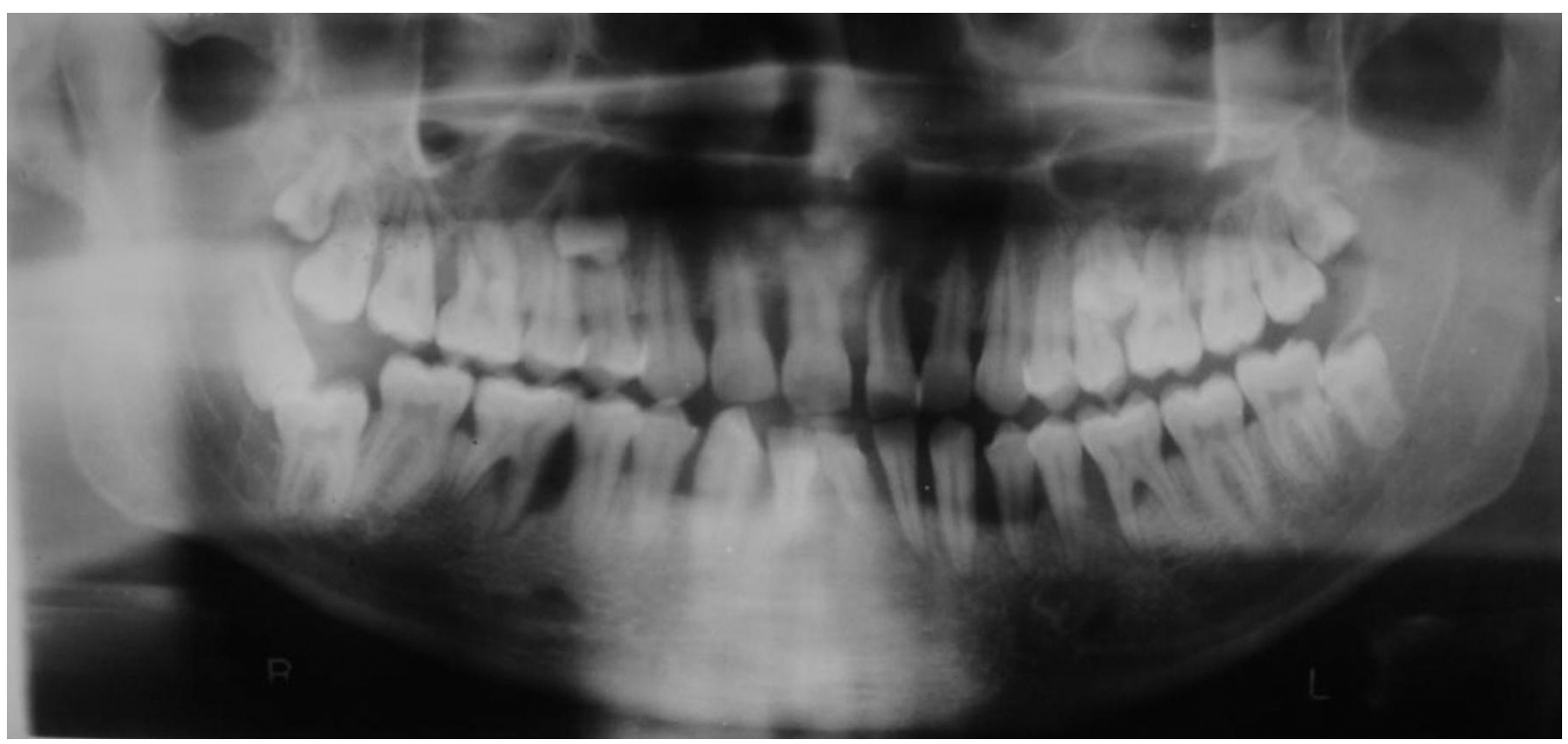

Figure 2. Pretreatment panoramic radiography showing periodontal bone loss and six impacted supernumerary teeth; 2 supplemental premolars and 4 disto-molars. 
resolution of the periodontal infection, the patient was placed on a personalized maintenance care program. He is under periodontal maintenance for the past 2 years (Figure 3 and 4). The patient was also informed about the presence of multiple impacted supernumerary teeth, the possible complications, and modalities of treatment. At present, the patient has chosen to remain under observation.

\section{DISCUSSION}

Aggressive periodontitis comprises 3 diseases-prepubertal, juvenile, and rapidly progressive periodontitis-that were formerly classified as early onset periodontitis lalso called periodontosis). Clinical forms of aggressive periodontitis include localized and generalized variants that show specific clinical and laboratory features. The features of generalized aggressive periodontitis are as follows: (1) it usually affects persons who are under 30 years of age, but may also affect older persons; (2) it is characterized by a generalized interproximal attachment loss that affects at least 3

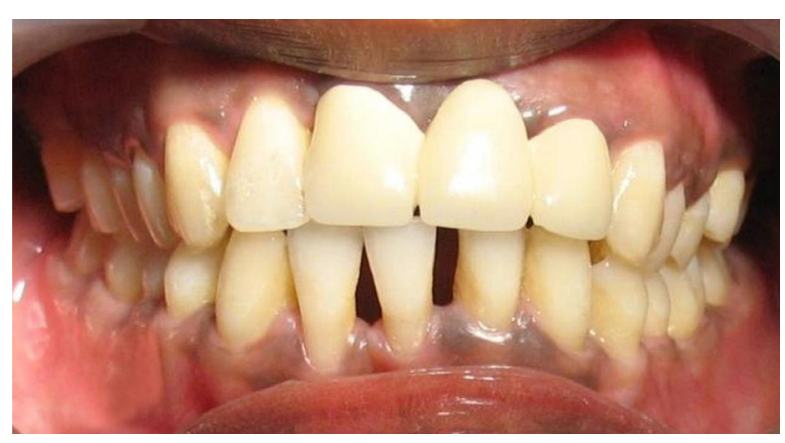

Figure 3. Clinical presentation after treatment showing gingiva in health. permanent teeth in addition to the first molars and incisors; (3) it is characterized by a pronounced episodic nature of the attachment and alveolar bone destruction; and (4) it leads to poor serum antibody response to infectious agents. ${ }^{5}$

According to the 1999 International Workshop for the Classification of Periodontal Disease, all the listed features need not be present for the diagnosis of aggressive periodontitis, and that clinical, radiological, and historical data alone can be used to differentiate aggressive periodontitis from chronic periodontitis. ${ }^{5}$ The diagnosis of aggressive periodontitis in our case was based on the following features: (1) noncontributory medical history, (2) rapid attachment loss and bone destruction, (3) inconsistency between microbial deposits and severity of destruction, (4) generalized interproximal attachment loss that affected at least 3 permanent teeth in addition to the first molars and incisors, and (5) radiographic finding of vertical bone defects.

Multiple supernumerary teeth are very rarely observed, and in most cases, they are associated with syndromes such as Gardner's syndrome, cleidocranial dysplasia, and cleft lip and palate. ${ }^{3}$ Many theories have been proposed to explain the genesis of supernumerary teeth, such as the theory of atavism or phylogenetic reversion, dichotomy of the tooth bud, and hyperactivity of the dental lamina. Of these, the theory of hyperactivity of the dental lamina seems the most acceptable. The exact etiology of the occurrence of supernumerary teeth is unknown, and both environmental and genetic basis have been suggested. The ge-

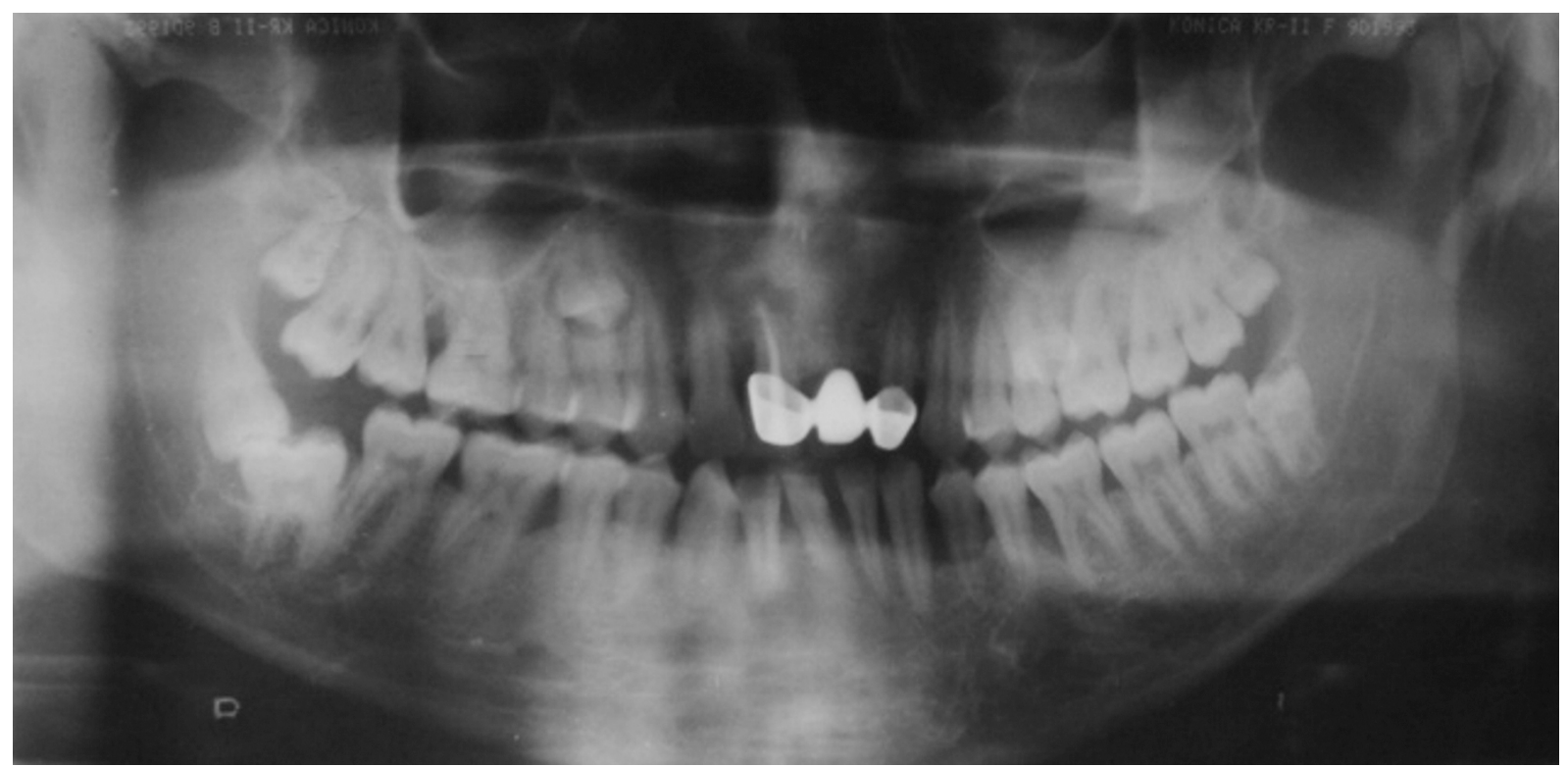

Figure 4. Post-treatment panoramic radiography showing bone fill in the vertical defects. 
Table 1. Data of studies that showed an association between aggressive periodontitis and supernumerary teeth.

\begin{tabular}{|c|c|c|c|c|c|c|c|}
\hline \multirow{2}{*}{ No. } & \multirow{2}{*}{ Author with reference number } & \multirow{2}{*}{$\begin{array}{l}\text { No. of } \\
\text { cases }\end{array}$} & \multirow{2}{*}{$\begin{array}{c}\text { Type of } \\
\text { periodontitis }\end{array}$} & \multirow[t]{2}{*}{ Gender } & \multicolumn{3}{|c|}{ Type of supernumerary teeth } \\
\hline & & & & & Anterior & Premolar & Molar \\
\hline 1 & Eley $(1974)^{7}$ & 1 & Periodontosis & Female & - & - & 4 \\
\hline \multirow{2}{*}{2} & \multirow{2}{*}{ Rubin et al $(1981)^{8}$} & \multirow{2}{*}{2} & \multirow{2}{*}{ Juvenile periodontosis } & Female & 1 & 3 & - \\
\hline & & & & Female & - & 2 & - \\
\hline 3 & $\begin{array}{l}\text { Mendieta and Gonzalez-Blanco } \\
\qquad(1988)^{9}\end{array}$ & 1 & Localized juvenile periodontitis & Female & - & 7 & - \\
\hline \multirow{2}{*}{4} & \multirow{2}{*}{ Odell and Hughes $(1995)^{4}$} & \multirow{2}{*}{2} & \multirow{2}{*}{ Localized juvenile periodontitis } & Female & - & 2 & - \\
\hline & & & & Female & - & 4 & - \\
\hline \multirow{3}{*}{5} & \multirow{3}{*}{ Açikgöz et al (2004) $)^{10}$} & \multirow{3}{*}{3} & \multirow{3}{*}{ Aggressive periodontitis } & Male & 3 & 2 & - \\
\hline & & & & Female & 1 & - & - \\
\hline & & & & Male & - & 2 & - \\
\hline 6 & Vikas et al (2010) ${ }^{11}$ & 1 & Aggressive periodontitis & Male & 1 & - & - \\
\hline
\end{tabular}

netic cause has been suggested on the basis of the fact that the prevalence of supernumerary teeth is higher among the relatives of the affected individuals. Clinical consequences of supernumerary teeth include crowding, ectopic and delayed eruption, diastema, rotation and displacement, cystic lesions, and resorption of the adjacent teeth. ${ }^{2}$ In the present case, 6 impacted supernumerary teeth were observed; 2 of these were premolars and the remaining 4 were distomolars. However, the patient's sibling and parents did not have any supernumerary teeth.

The association between aggressive periodontitis and supernumerary teeth has been shown in 6 studies, ${ }^{4,7-11}$ i.e., 5 case studies and 1 retrospective study. The association between juvenile periodontitis and multiple supernumerary teeth was first shown by Kaslick and Chasens in $1968^{\circ}$ in their study that included 27 black patients. However, Eley reported an acceptable case of periodontosis and supernumerary teeth 4 supernumerary molars). ${ }^{7}$ Since then, other researchers ${ }^{4,8-11}$ have also reported such a phenomenon (Table 1).

Aggressive periodontitis and supernumerary teeth have a familial pattern of inheritance and racial predisposition. ${ }^{4,10}$ However, aggressive periodontitis has not been observed in patients with any of the syndromes that are typically characterized by the presence of multiple supernumerary teeth. Environmental factors act as etiological factors for aggressive periodontitis and supernumerary teeth. ${ }^{10}$ Thus, both these entities exhibit etiological heterogeneity. Hence, a concomitant presentation of aggressive periodontitis and supernumerary teeth may have a genetic basis or could be a chance occurrence. ${ }^{4,10}$ Notably, both these conditions also show genetic heterogeneity, in that different genetic mechanisms may play a role in their etiology. ${ }^{1,12,13}$ Therefore, determination of the genetic basis for this association is very difficult

\section{CONCLUSION}

In conclusion, we report the nonsyndromic case of multiple impacted supernumerary teeth and generalized aggressive periodontitis. However, in the future, large population studies are needed to determine the type of relationship that may exist between these 2 conditions.

\section{REFERENCES}

1. Tonetti MS, Mombelli A. Aggressive periodontitis. In, Lindhe J, Lang NP, Karring T (ed). Clinical Periodontology and Implant Dentistry, $5^{\text {th }}$ ed. USA, Blackwell Munksgaard, 2008; 428-458.

2. Ashish S, Daljit SG, Christopher T, Farhad BN. Diagnosis and management of supernumerary teeth. Dent Update 2008;35:510-520.

3. Golan I, Baumert U, Hrala BP, Müssig D. Early craniofacial signs of Cleidocranial dysplasia. Int J Paediatr Dent 2004;14:49-53.

4. Odell EW, Hughes FJ. The possible association between localized juvenile periodontitis and supernumerary teeth. $J$ Periodontol 1995;66:449-451.

5. Lang NP, Bartold PM, Cullinan M, Jeffcoat M, Mombelli A, Murakami S, et al. International classification workshop. Consensus report: Aggressive periodontitis. Ann Periodontol 1999;4:53.

6. Kaslick RS, Chasens Al. Periodontosis with periodontitis: a study involving young adult males.II.Clinical, medical, and histopathologic studies. Oral Surg 1968;25:327-350. 
7. Eley BM. Periodontosis occurring with supernumerary molars. J Dent 1974;2:167-170.

8. Rubin MM, Nevins A, Berg M, Borden B. A comparison of identical twins in relation to three dental anomalies: multiple supernumerary teeth, juvenile periodontosis, and zero caries incidence. Oral Surg Oral Med Oral Pathol 1981;52:391-394

9. Mendieta C, Gonzalez-Blanco A. Supernumerary teeth and localized juvenile periodontitis: a review of the literature and case report. Periodontal Case Rep 1988;10:23-27.

10. Açikgöz G, Açikgöz A, Keskiner I, Türk T, Otan F. Aggressive periodontitis with supernumerary teeth: a retrospective study. J Periodontol 2004;75:1458-1460.

11. Vikas V, Amit G, Sabir M. Supernumerary eumorphic mandibular incisor in association with aggressive periodontitis. J Indian Soc Periodontol 2010;2:136-138.

12. Batra P, Duggal R, Prakash H. Non-syndromic multiple supernumerary teeth transmitted as an autosomal dominant trait. J Oral Pathol Med 2005;34:621-625.

13. Pavithra S, Aravind N. Mesiodens with an unusual morphology and multiple impacted supernumerary teeth in a non-syndromic patient. Indian J Dent Res 2007;18:138-140. 